\title{
МЕТОДЫ ИЗМЕРЕНИЯ КОНЦЕНТРАЦИИ ДНК: СОВПАДЕНИЕ ОТНОСИТЕЛЬНЫХ ВЕЛИЧИН И РАЗЛИЧИЯ АБСОЛЮТНЫХ
}

О. П. Балановский

${ }^{1}$ Институт общей генетики имени Н. И. Вавилова, Москва, Россия

${ }^{2}$ Медико-генетический научный центр, Москва, Россия

${ }^{3}$ Биобанк Северной Евразии, Москва, Россия

Измерение концентрации ДНК является базовым методом, от надежности которого зависит эффективность дальнейшего хранения и использования образцов. Существует несколько широко распространенных и хорошо зарекомендовавших себя способов измерения концентрации ДНК, однако степень их согласованности друг с другом изучена недостаточно. Целью работы было измерить концентрации одних и тех же образцов разными методами и провести сравнительный анализ полученных результатов. В двух независимых экспериментах, суммарно включивших 100 образцов геномной ДНК, сравнивали три метода определения концентрации ДНК: спектрофотометрический (Nanodrop), флуориметрический (Qubit) и ПЦР в реальном времени (Quantifiler). Выявлено, что значения концентрации ДНК, полученные разными методами, хорошо коррелируют друг с другом (коэффициенты корреляции составляют 0,98-0,99). Однако при такой отличной корреляции относительных величин концентрации абсолютные величины, полученные разными методами, варьируют значительно, вплоть до двукратных различий. Спектрофотометрический метод дает наиболее высокие концентрации, ПЦР в реальном времени - промежуточные, а флуориметрический — наиболее низкие. Различия в результатах более выражены для образцов с низкой концентрацией. Мы рекомендуем учитывать наличие этих систематических различий между результатами измерения концентрации ДНК, полученными разными методами.

Ключевые слова: концентрация ДНК, методы измерения, ПЦР в реальном времени, спектрофотометрический метод, флуориметрический метод

Финансирование: исследование выполнено в рамках Государственного задания для Медико-генетического научного центра (эксперимент 1) и при поддержке гранта РНФ 17-14-01345 (эксперимент 2).

Информация о вкладе авторов: О. П. Балановский - дизайн исследования, анализ данных, написание текста статьи; Ж. А. Кагазежева — проведение экспериментальных работ; М. В. Олькова — проведение экспериментальных работ, подбор литературы.

Соблюдение этических стандартов: исследование выполнено на образцах, полученных в ходе популяционно-генетических обследований генофонда и одобрено Этическим комитетом Медико-генетического научного центра (протокол № 3/1 от 5 сентября 2018 г.). Все участники исследования подписали добровольное информированное согласие на участие в исследовании и публикацию данных.

$\triangle$ Для корреспонденции: Олег Павлович Балановский

ул. Губкина, д. 3, г. Москва, 119991; balanovsky@inbox.ru

Статья получена: 21.06.2019 Статья принята к печати: 27.06.2019 Опубликована онлайн: 30.06.2019

DOI: 10.24075/vrgmu.2019.043

\section{METHODS FOR DNA QUANTIFICATION YIELD SIMILAR RELATIVE BUT DIFFERENT ABSOLUTE VALUES}

Balanovsky OP ${ }^{1,2,3 凶}$, Kagazezheva ZhA ${ }^{1,2}$, Olkova MV²

${ }^{1}$ Vavilov Institute of General Genetics, Moscow, Russia

${ }^{2}$ Research Center for Medical Genetics, Moscow, Russia

${ }^{3}$ Biobank of North Eurasia, Moscow, Russia

DNA quantification is a routine yet important procedure that determines the efficacy of long-term sample storage and further manipulations with the sample. There are a few well-established methods for measuring DNA concentrations. However, it still not fully clear how concordant their results are. The aim of this work was to measure DNA concentrations in a set of samples using different quantification methods and to compare the obtained values. In 2 independent experiments, a total of 100 genomic DNA samples were analyzed using 3 different DNA quantification methods, including spectrophotometry (NanoDrop), fluorometry (Qubit) and real-time PCR (Quantifiler). The obtained relative concentrations demonstrated an excellent correlation (the correlation coefficients were as high as 0.98 to 0.99 ). However, the absolute concentrations showed a considerable variation and even a twofold difference. Spectrophotometry yielded the highest concentrations, whereas fluorometry yielded the lowest. The real-time PCR results were intermediate. The differences were more pronounced for the samples with low DNA concentrations. We recommend that such differences should be accounted for when estimating DNA concentrations using an arsenal of different quantification methods.

Keywords: DNA concentration, measurement method, real-time PCR, spectrophotometry, fluorometry

Funding: this study was part of the Government contract with the Research Center for Medical Genetics (Experiment 1). Experiment 2 was supported in part by Grant 17-14-01345 of the Russian Science Foundation.

Author contribution: Balanovsky OP conceived the study, analyzed the obtained data and wrote this manuscript; Kagazezheva ZhA conducted one of the experiments; Olkova MV conducted one of the experiments and analyzed the literature.

Compliance with ethical standards: the study was approved by the Ethics Committee of the Research Center for Medical Genetics (Protocol № 3/1 dated september 5, 2018). The analyzed samples were obtained during population genetic studies. All study participants gave informed consent to participate.

$\measuredangle$ Correspondence should be addressed: Oleg P. Balanovsky

Gubkina 3, Moscow, 119991; balanovsky@inbox.ru

Received: 21.06.2019 Accepted: 27.06.2019 Published online: 30.06.2019

DOI: 10.24075/brsmu.2019.043

Измерение концентрации ДНК является базовым рутинным методом, используемым во многих лабораториях, и полученные значения используют в последующей работе с образцами ДНК - от долгосрочного хранения и биобанкирования до использования в NGS.
Существует несколько широко распространенных и хорошо зарекомендовавших себя способов измерения концентрации ДНК, удобство использования которых обусловлено в том числе наличием коммерчески доступных наборов и специализированных приборов различных 
производителей. Во многих лабораториях сталкивались с тем, что значения концентрации ДНК, полученные разными методами, порой плохо согласуются, а существующие по данному вопросу литературные источники [1-6] указывают на то, что разные методы имеют ограничения и особенности применения, которые не всегда изучены. Массовые измерения концентрации геномной ДНК и геномных библиотек проводят, например, перед проведением полногеномного секвенирования, а во многих биобанках - при принятии образцов и мониторинге их сохранности. Выбор оптимального метода измерения концентрации ДНК, выявление его особенностей и сопоставимости с другими методами являются одной из задач, стоящих перед нашим коллективом в процессе работы с обширными коллекциями Биобанка Северной Евразии [7], а также при выполнении ряда популяционных проектов. Можно предположить, что сходные задачи могут стоять и перед многими другими лабораториями. Целью работы было измерить концентрации одних и тех же образцов разными методами и провести сравнительный анализ полученных результатов: оценить воспроизводимость каждого метода; определить степень сходства результатов, полученных разными методами; выявить закономерности в различиях между результатами, полученными этими тремя методами.

\section{МАТЕРИАЛЫ И МЕТОДЫ}

В течение 2017-2018 гг. мы провели ряд экспериментов, сравнивая результаты измерений концентрации ДНК, полученные разными методами на одних и тех же образцах. Использовали три наиболее широко распространенных метода определения концентрации ДНК: а) спектрофотометрический метод, позволяющий оценить качество и количество ДНК по уровню поглощения образцом волн определенной длины с помощью Nanodrop; б) фрлуориметрический метод с использованием флуоресцентных красителей (Qubit); в) ПЦР в реальном времени (Human DNA Quantifiler).

Все эксперименты демонстрировали сходную картину, но в данной статье для краткости будут приведены результаты только двух из них, выполненных наиболее тщательно и с контролем наибольшего числа параметров: эксперимента 1 и эксперимента 2. Оба эксперимента проведены на популяционных образцах, хранящихся в Биобанке Северной Евразии и выделенных фенолхлороформным методом.

В эксперименте 1 использовали 49 образцов ДНК. Концентрация образцов была предварительно оценена с помощью Qubit и для высококонцентрированных образцов уменьшена до значений, не превышающих 50 нг/Мкл, поскольку набор Human Quantifiler (при использовании внутренних стандартов в строгом соответствии с инструкцией производителя) гарантирует точные измерения при концентрациях до 55 нг/мкл. Для этих образцов проводили измерение концентрации ДНК методом спектрофотометрии с помощью Nanodrop (в трех повторностях для каждого образца), методом флуориметрии с помощью Qubit (в трех повторностях) и методом ПЦР в реальном времени с помощью Human DNA Quantifiler (в связи с высокой стоимостью реактивов этот эксперимент проводили для половины образцов в двух повторностях, и поскольку корреляция между ними составила 0.99, для второй половины образцов провели в одной повторности).
Спектрофотометрические измерения осуществляли на приборе NanoDrop 2000 (Thermo Fisher Scientific; США) в строгом соответствии с инструкциями производителя. Забор образца осуществляли автоматической пипеткой объемом 1-10 мкл Research Plus (Eppendorf; Германия). Концентрацию ДНК измеряли в образце объемом 2 мкЛ при температуре $20^{\circ} \mathrm{C}$.

Флуориметрические измерения проводили на приборе Qubit 4 (Thermo Fisher Scientific; США). При работе с прибором использовали тонкостенные пробирки для

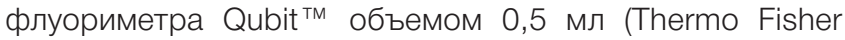
Scientific; США). Концентрацию ДНК измеряли с помощью набора Qubit ${ }^{\mathrm{T}}$ dsDNA BR Assay Kit (Thermo Fisher Scientific; США) в строгом соответствии с инструкциями производителя [7]. Забор образцов осуществляли автоматической пипеткой Research Plus объемом 0,1-2,5 мкл (Eppendorf; Германия). Концентрацию ДНК измеряли в образце объемом 2 мкл при температуре $20^{\circ} \mathrm{C}$.

Измерение концентрации методом ПЦР в реальном времени проводили на приборе 7500 Real-Time PCR System for Human Identification (Applied Biosystems; США) с использованием набора Quantifiler ${ }^{\text {тм }}$ Human DNA Quantification Kit (Thermo Fisher Scientific; CШA) B строгом соответствии с инструкциями производителя. Для амплификации использовали по 2 мкл образца ДНК. Забор образца осуществляли автоматической пипеткой Research Plus объемом 0,1-2,5 мкл (Eppendorf; Германия).

В эксперименте 2 использовали 51 образец ДНК, выделенной фенол-хлорофрормным методом. Концентрацию ДНК во всех образцах также измеряли с помощью Nanodrop, Qubit и Quantifiler. Измерения с помощью Qubit проводили в двух повторностях, измерения остальными методами - в одной повторности. Диапазон концентраций ДНК, предварительно оцененный с помощью Qubit, в эксперименте 2 был сдвинут в большую сторону по сравнению с экспериментом 1 - от 20 до 90 нг/мкл.

Эксперименты 1 и 2 проводили разные операторы в разные периоды времени.

Первичную обработку данных и построение графиков проводили в MS Excel, расчет коэффиициентов корреляций в программе Statistica 7.

\section{РЕЗУЛЬТАТЫ ИССЛЕДОВАНИЯ}

Результаты эксперимента 1 представлены в табл. 1, эксперимента 2 - в табл. 2. Эксперименты однотипны, поэтому их результаты представлены на рисунке и в табл. 3 и 4 параллельно. Сначала описаны результаты более подробного эксперимента 1, а затем дано их сравнение с итогами эксперимента 2.

Прежде всего мы оценивали воспроизводимость результатов эксперимента 1 в разных повторностях для одного и того же образца, полученных одним методом. Она оказалась чрезвычайно высокой (коэффициент корреляции варьировал от 0,99 до 1,00), что свидетельствует по крайней мере об отсутствии эффекта погрешностей пипетирования в эксперименте 1. Поэтому в дальнейшем мы использовали для каждого образца средние значения его концентрации по всем повторностям.

Метод оценки концентрации, основанный на ПцР в реальном времени (далее - Quantifiler), считают наиболее точным, поэтому мы взяли его в качестве условного стандарта и сравнили с ним результаты остальных двух методов (выбор другого стандарта не изменил бы выводов). На рис. 1А по оси абсцисс отложены концентрации, 
полученные методом ПЦР в реальном времени, а по оси ординат - значения концентраций, полученные всеми тремя методами. В этом случае график для Quantifiler может представлять собой только прямую под углом 45 (черная линия), которая служит «линией отсчета», но поведение графиков двух других методов представляет интерес.

таблица 1. Результаты измерений концентрации в отдельных образцах в эксперименте 1

\begin{tabular}{|c|c|c|c|c|c|c|c|c|}
\hline SampleID & Quantifiler1 & Quantifiler2 & Qubit1 & Qubit2 & Qubit3 & Nanodrop1 & Nanodrop2 & Nanodrop3 \\
\hline 21 & 10,7 & 10,3 & 6,1 & 5,9 & 5,6 & 11,6 & 13,1 & 12,0 \\
\hline 22 & 18,3 & 17,6 & 12,2 & 11,2 & 11,4 & 23,7 & 25,0 & 24,1 \\
\hline 23 & 36,6 & 33,2 & 24,4 & 24,1 & 24,0 & 51,6 & 51,2 & 49,8 \\
\hline 24 & 44,2 & 47,8 & 34,0 & 36,2 & 31,9 & 73,7 & 75,2 & 73,2 \\
\hline 25 & 64,3 & 58,4 & 47,2 & 45,0 & 49,6 & 101,1 & 104,4 & 102,8 \\
\hline 31 & 10,2 & 9,8 & 5,5 & 5,4 & 5,6 & 8,6 & 7,9 & 8,0 \\
\hline 32 & 18,1 & 18,3 & 11,2 & 11,0 & 10,9 & 15,7 & 16,4 & 17,1 \\
\hline 33 & 37,0 & 35,7 & 23,6 & 24,5 & 23,4 & 38,6 & 37,6 & 39,5 \\
\hline 34 & 44,7 & 47,4 & 32,9 & 30,1 & 31,4 & 51,5 & 54,4 & 51,7 \\
\hline 35 & 59,2 & 54,4 & 42,1 & 43,3 & 42,6 & 67,8 & 70,7 & 69,4 \\
\hline 41 & 10,3 & 9,7 & 5,6 & 6,1 & 5,2 & 8,2 & 7,7 & 7,5 \\
\hline 42 & 14,8 & 15,5 & 11,6 & 11,0 & 10,9 & 16,3 & 16,9 & 16,2 \\
\hline 43 & 28,7 & 27,3 & 22,3 & 23,0 & 22,7 & 35,8 & 35,8 & 36,8 \\
\hline 44 & 40,1 & 39,8 & 32,8 & 32,3 & 33,5 & 53,2 & 51,5 & 53,4 \\
\hline 45 & 59,0 & 55,3 & 45,3 & 45,7 & 46,0 & 70,7 & 71,9 & 71,7 \\
\hline 51 & 10,1 & 10,5 & 5,9 & 5,7 & 5,5 & 10,0 & 9,3 & 9,3 \\
\hline 52 & 20,1 & 20,2 & 11,8 & 11,6 & 11,5 & 19,9 & 19,2 & 19,5 \\
\hline 53 & 34,2 & 35,8 & 23,2 & 22,0 & 23,4 & 38,4 & 39,4 & 36,3 \\
\hline 54 & 47,7 & 44,6 & 31,9 & 33,7 & 34,0 & 59,5 & 57,8 & 58,1 \\
\hline 71 & 9,0 & 8,8 & 5,3 & 5,4 & 5,1 & 8,1 & 8,1 & 8,4 \\
\hline 72 & 15,5 & 15,4 & 9,7 & 10,3 & 10,3 & 17,2 & 17,5 & 17,7 \\
\hline 73 & 28,2 & 30,6 & 18,5 & 19,5 & 19,1 & 36,3 & 36,1 & 36,3 \\
\hline 74 & 42,3 & 44,6 & 34,2 & 27,7 & 30,3 & 54,3 & 52,7 & 54,1 \\
\hline 75 & 53,0 & 58,1 & 40,6 & 46,3 & 41,9 & 75,2 & 72,1 & 72,6 \\
\hline 81 & 10,8 & & 5,8 & 5,7 & 5,8 & 9,1 & 9,0 & 9,0 \\
\hline 82 & 23,1 & & 11,8 & 11,6 & 11,8 & 20,4 & 19,3 & 19,5 \\
\hline 83 & 32,7 & 32,4 & 21,9 & 21,7 & 21,2 & 36,5 & 37,5 & 36,8 \\
\hline 84 & 48,3 & & 32,0 & 33,6 & 33,9 & 59,7 & 58,0 & 58,3 \\
\hline 85 & 63,6 & & 45,6 & 46,9 & 47,0 & 77,1 & 80,5 & 80,5 \\
\hline 91 & 10,9 & & 5,7 & 5,8 & 5,8 & 10,2 & 9,8 & 9,8 \\
\hline 92 & 19,4 & & 11,7 & 11,9 & 10,9 & 22,4 & 23,3 & 23,5 \\
\hline 93 & 33,9 & & 22,2 & 22,9 & 23,0 & 46,2 & 47,0 & 46,0 \\
\hline 94 & 51,5 & & 36,0 & 35,5 & 36,0 & 70,3 & 70,9 & 69,6 \\
\hline 95 & 62,2 & & 40,3 & 45,3 & 46,6 & 92,3 & 90,2 & 91,5 \\
\hline 101 & 10,1 & & 5,6 & 5,9 & 5,7 & 10,7 & 10,5 & 11,6 \\
\hline 102 & 22,4 & & 12,5 & 12,3 & 12,2 & 23,8 & 24,6 & 21,9 \\
\hline 103 & 39,0 & & 22,0 & 23,3 & 22,8 & 50,3 & 50,7 & 50,0 \\
\hline 104 & 51,9 & & 32,2 & 34,0 & 34,1 & 70,4 & 70,6 & 68,0 \\
\hline 105 & 63,0 & & 44,7 & 46,4 & 46,4 & 95,0 & 92,0 & 95,5 \\
\hline 111 & 8,3 & & 4,5 & 4,7 & 4,7 & 7,0 & 7,5 & 7,7 \\
\hline 112 & 17,4 & & 10,1 & 10,3 & 10,0 & 14,9 & 14,9 & 16,3 \\
\hline 113 & 30,2 & & 18,0 & 19,6 & 20,2 & 31,5 & 33,0 & 29,8 \\
\hline 114 & 43,6 & & 28,8 & 28,3 & 30,0 & 45,6 & 44,6 & 46,2 \\
\hline 115 & 56,9 & & 39,3 & 41,8 & 40,5 & 62,8 & 63,3 & 61,4 \\
\hline 121 & 9,8 & & 5,3 & 5,1 & 4,8 & 10,3 & 9,9 & 10,4 \\
\hline 122 & 20,5 & & 10,4 & 10,9 & 11,2 & 21,0 & 21,8 & 21,9 \\
\hline 123 & 36,2 & & 22,3 & 22,6 & 23,5 & 43,9 & 43,7 & 42,4 \\
\hline 124 & 49,4 & & 32,6 & 32,3 & 32,0 & 63,0 & 64,2 & 63,9 \\
\hline 125 & 56,4 & & 41,9 & 41,5 & 42,8 & 84,0 & 82,8 & 81,9 \\
\hline
\end{tabular}


Таблица 2. Результаты измерений концентрации в отдельных образцах в эксперименте 2

\begin{tabular}{|c|c|c|c|c|}
\hline SampleID & Nanodrop & Qubit1 & Qubit2 & Quantifiler \\
\hline 1 & 67,5 & 45,4 & 50,2 & 56,0 \\
\hline 2 & 71,9 & 51,2 & 52 & 56,2 \\
\hline 3 & 99,1 & 78 & 65,1 & 78,5 \\
\hline 4 & 59,2 & 47,5 & 49 & 48,4 \\
\hline 8 & 97,1 & 69,7 & 76,2 & 83,7 \\
\hline 10 & 76,4 & 67,2 & 65,9 & 68,1 \\
\hline 11 & 58 & 46,7 & 43,3 & 46,6 \\
\hline 12 & 88,7 & 66,8 & 63,7 & 73,1 \\
\hline 13 & 59,8 & 42,2 & 40,8 & 48,9 \\
\hline 14 & 92,4 & 62,1 & 66,1 & 79,8 \\
\hline 15 & 78,6 & 50,4 & 51,8 & 62,7 \\
\hline 16 & 74,3 & 50,7 & 51,4 & 60,5 \\
\hline 17 & 72,5 & 29,6 & 48,4 & 43,6 \\
\hline 18 & 47,5 & 34,6 & 35,5 & 39,0 \\
\hline 19 & 84,5 & 59,1 & 59,8 & 63,7 \\
\hline 20 & 98,5 & 71,2 & 77 & 83,5 \\
\hline 21 & 69,9 & 56,4 & 61,3 & 68,5 \\
\hline 26 & 66,2 & 45,2 & 33,8 & 62,3 \\
\hline 27 & 100,1 & 54,9 & 77,9 & 81,3 \\
\hline 28 & 97 & 74,1 & 75 & 79,5 \\
\hline 29 & 95,6 & 62,8 & 68,1 & 72,4 \\
\hline 32 & 58,5 & 39,2 & 40,6 & 41,5 \\
\hline 33 & 71,3 & 47,4 & 44,3 & 61,1 \\
\hline 34 & 82,6 & 43 & 64,1 & 75,3 \\
\hline 35 & 101,7 & 69,2 & 74,1 & 86,5 \\
\hline 37 & 101,6 & 73,3 & 74 & 84,5 \\
\hline 44 & 101,7 & 49,8 & 48,3 & 70,2 \\
\hline 46 & 99,2 & 86 & 63,6 & 93,9 \\
\hline 48 & 101,7 & 70,7 & 78,5 & 80,1 \\
\hline 49 & 50,9 & 36,5 & 37,5 & 41,2 \\
\hline 51 & 50,8 & 27,4 & 29,8 & 34,8 \\
\hline 52 & 48,1 & 38,7 & 42,2 & 50,3 \\
\hline 53 & 52,6 & 31,9 & 46,1 & 54,4 \\
\hline 54 & 27,2 & 18,3 & 15,9 & 23,1 \\
\hline 55 & 52,5 & 34,6 & 36,8 & 36,7 \\
\hline 56 & 55,8 & 32,7 & 34,7 & 72,3 \\
\hline 57 & 50,2 & 31,2 & 35,8 & 50,1 \\
\hline 58 & 62,7 & 58,2 & 50,9 & 28,5 \\
\hline 59 & 36,3 & 23,2 & 26,7 & 25,7 \\
\hline 60 & 18,5 & 11,4 & 11,9 & 31,9 \\
\hline 61 & 34,8 & 22,2 & 25,9 & 27,1 \\
\hline 62 & 73,3 & 28,2 & 55,9 & 66,6 \\
\hline 63 & 112,5 & 50 & 90,2 & 92,2 \\
\hline 65 & 54,1 & 21,7 & 36 & 42,3 \\
\hline 72 & 63,3 & 42,9 & 50,6 & 53,4 \\
\hline 73 & 66,9 & 40,1 & 36,5 & 50,0 \\
\hline 74 & 68,9 & 40,2 & 47,4 & 50,4 \\
\hline 75 & 41,1 & 25,4 & 24,7 & 29,1 \\
\hline 76 & 75 & 43,3 & 51,4 & 63,8 \\
\hline 77 & 71,7 & 51,1 & 50,4 & 61,2 \\
\hline 79 & 99,7 & 64,8 & 64,9 & 74,9 \\
\hline
\end{tabular}


A

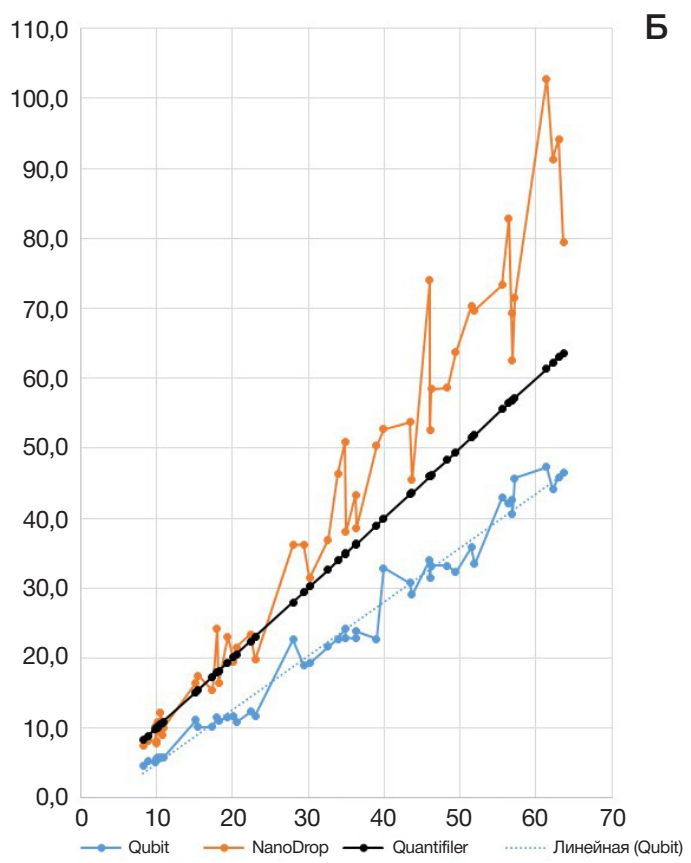

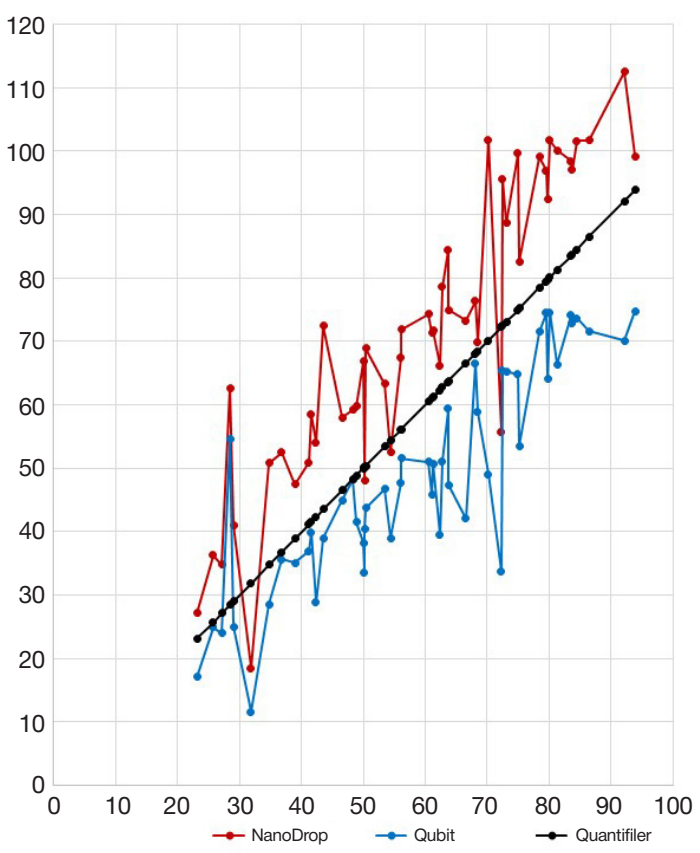

Рис. 1. Сравнение концентраций ДНК, полученных разными методами, для отдельных образцов в эксперименте 1 (А); в эксперименте 2 (Б). По оси абсцисс: значения концентрации, полученные с помощью Quantifiler. По оси ординат: значения концентрации, полученные с помощью Quantifiler (черная линия), Qubit (синяя линия) и Nanodrop (красная линия)

Грасрик для Nanodrop (рис. 1A; красная линия) построен на значениях, в целом сходных с Quantifiler, но они несколько более высокие и со стохастическими колебаниями («биения» графика). Отметим, что завышение результатов, полученных с помощью Nanodrop, по сравнению с результатами Quantifiler было отмечено для образцов с концентрациями выше 20 нг/мкл, а для более низких концентраций результаты двух методов выглядят чрезвычайно близкими.

Если красный график (Nanodrop) расположен выше черной линии (Quantifiler), то синий график (Qubit) - ниже ее. Действительно, для всех образцов концентрации, измеренные с помощью Qubit, оказываются ниже концентраций, полученных с помощью Quantifiler. При сравнении с Nanodrop результаты Qubit выглядят более устойчивыми (график более ровный). Хотя на первый взгляд создается впечатление, что график Qubit просто смещен вниз (результаты занижены на фиксированную величину), но линия тренда Qubit (синяя пунктирная линия) показывает, что это не так: график результатов Qubit отличается от графика результатов Quantifiler не только систематическим занижением, но и тангенсом угла наклона.

После рассмотрения результатов на качественном уровне перейдем к количественному сравнению. В табл. 3 (значения над диагональю) представлены коэффициенты корреляции между результатами, полученными всеми тремя методами. Эти коэффициенты оказались велики (не ниже 0,98), демонстрируя отличную корреляцию между концентрациями, оцененными разными методами. Однако при сравнении средних величин картина уже иная (табл. 4). Прежде всего, концентрации, измеренные

Таблица 3. Коэффициенты корреляции между значениями концентрации, полученными разными методами. Над диагональю: эксперимент 1. Под диагональю: эксперимент 2

\begin{tabular}{|c|c|c|c|}
\hline & Quantifiler & Qubit & Nanodrop \\
\hline Quantifiler & 1 & 0,99 & 0,98 \\
\hline Qubit & 0,93 & 1 & 0,98 \\
\hline Nanodrop & 0,91 & 0,88 & 1 \\
\hline
\end{tabular}

Таблица 4. Средние значения концентрации, полученные разными методами

\begin{tabular}{|c|c|c|c|c|c|c|}
\hline & & Quantifiler & Qubit & Nanodrop & Qubit/Quantifiler & Nanodrop/Quantifiler \\
\hline \multirow{8}{*}{ Эксперимент 1} & Все образцы & 33,1 & 22,7 & 41,3 & 0,68 & 1,25 \\
\hline & lower conc $(n=24)$ & 17,0 & 10,4 & 18,0 & 0,61 & 1,05 \\
\hline & higher conc $(n=25)$ & 48,6 & 34,5 & 63,8 & 0,71 & 1,31 \\
\hline & conc_range1 $(n=10)$ & 9,9 & 5,5 & 9,3 & 0,55 & 0,94 \\
\hline & conc_range2 $(n=10)$ & 19,0 & 11,2 & 19,7 & 0,59 & 1,04 \\
\hline & conc_range3 $(n=10)$ & 33,6 & 22,2 & 40,8 & 0,66 & 1,22 \\
\hline & conc_range4 $(n=10)$ & 46,6 & 32,6 & 59,9 & 0,70 & 1,29 \\
\hline & conc_range5 $(n=10)$ & 59,2 & 44,2 & 80,8 & 0,75 & 1,36 \\
\hline Эксперимент 2 & Все образцы & 59,0 & 49,2 & 71,4 & 0,83 & 1,21 \\
\hline
\end{tabular}


с помощью Qubit, оказываются значительно (примерно в полтора раза) ниже концентраций, измеренных с помощью Quantifiler, а измеренные с помощью Nanodrop - наоборот, на 25\% выше концентраций Quantifiler. Более того, степень выраженности различий в концентрациях сама зависит от концентрации: когда выборка была поделена нами на две подвыборки равного объема, но разной средней концентрации, то соотношение средних концентраций Qubit/Quantifiler составило 61\% для образцов с низкими концентрациями и 71\% для образцов с более высокими концентрациями (табл. 4; различия, согласно критерию Манна-Уитни, достоверны при $p=0,01)$. Чтобы исследовать закономерность зависимости расхождения измерений, сделанных разными методами, от концентрации ДНК более подробно, мы разделили нашу выборку на 5 подвыборок по степени возрастания концентрации и рассчитали соотношение концентраций Qubit/Quantifiler (табл. 4). Несмотря на малый объем подвыборок (по 10 образцов), была выявлена четкая закономерность: соотношение концентраций, полученных двумя методами, монотонно увеличивается от 55\% (т. е. двукратных различий при низких концентрациях) до 75\% (т. е. различий на четверть при высоких концентрациях).

Все описанные результаты относились к эксперименту 1. Что касается эксперимента 2, то график индивидуальных образцов (рис. 1Б) демонстрирует ту же закономерность: по сравнению с оценкой концентрации, полученной методом ПЦР в реальном времени (Quantifiler), Nanodrop дает завышенные концентрации (красный график), а Qubit - заниженные (синий график). Обращает на себя внимание, что статистический шум (хаотичность грасиков) выражен в эксперименте 2 намного сильнее, что мы относим к «эффекту оператора». Количественный анализ результатов эксперимента 2, как и для эксперимента 1, выявляет высокую корреляцию результатов, полученных разными методами. Но эффект меньшего опыта работы оператора в эксперименте 2 проявляется и в этом случае: коэфффициенты корреляции, хотя и высоки $(0,88-0,93)$, все же явно ниже, чем в эксперименте 1 (табл. 3; значения под диагональю). Сравнение средних значений, как и в эксперименте 1, выявило значительное расхождение между методами: Qubit на 20\% занижает, a Nanodrop на 20\% завышает концентрации по сравнению с Quantifiler. Единственное, что не было выявлено в эксперименте 2, зависимость соотношения концентраций, оцененных разными методами, от самой концентрации. Мы относим это к большей экспериментальной погрешности в эксперименте 2: в результате «эффекта оператора» эта закономерность не проявляется при данном объеме выборки.

\section{ОБСУЖДЕНИЕ РЕЗУЛЬТАТОВ}

Оба эксперимента, проведенные в разное время разными операторами с разным опытом работы и на разных образцах, выявили одинаковые закономерности: концентрации ДНК, измеренные разными методами, хорошо скоррелированы друг с другом, но значительно различаются по оцениваемой ими величине концентрации ДНК. Иными словами, разные методы определения концентрации ДНК дают хорошую согласованность относительных, но резкое различие абсолютных величин концентрации ДНК. Более того, чем ниже концентрации, тем более существенными становятся эти различия между методами. Это означает, что относительные значения концентрации, полученные любым из трех использованных методов, достаточно надежны и могут использоваться для сравнения с другими образцами, но эти образцы обязательно должны быть измерены тем же самым методом. А вот совместное использование в одном исследовании значений концентрации, полученных разными методами, представляет собой проблему. Можно было бы предложить коэффициенты пересчета (значения последних двух столбцов в табл. 4 могут выступать в этой роли), но такой пересчет будет сложным, поскольку коэффициент пересчета сам зависит от концентрации.

В ранее проведенных работах, посвященных сравнению методов измерения концентрации ДНК, были выявлены как согласованность [1], так и различия между разными методами [3, 4]. Особенностью нашего исследования стало использование большого числа образцов (суммарно в обоих экспериментах их 100, тогда как в большинстве аналогичных работ - 3-6 образцов), что позволило провести статистический анализ и выявить зависимость степени согласованности методов от концентрации.

Отметим, что, исходя из особенностей методов, можно было ожидать разное соотношение. С одной стороны, значения, полученные методом ПЦР в реальном времени (Quantifiler), могут быть ниже полученных флуориметрическим методом (Qubit), поскольку ПЦР в реальном времени оценивает только эффективную концентрацию ДНК (ДНК, вступающую в ПЦР, т. е. фрагменты достаточной длины и без повреждений), тогда как Qubit оценивает всю двухцепочечную ДНК. С другой стороны, значения, полученные методом ПЦР в реальном времени, могут быть выше полученных флуориметрически, так как в процесс ПЦР вовлечены как двухцепочечные, так и одноцепочечные нити ДНК каждого из образцов, а фллуориметр получает информацию о концентрации лишь двухцепочечной ДНК в образце. Что касается завышенных концентраций ДНК при спектрофотометрическом измерении по сравнению с измерением концентрации методом ПЦР в реальном времени, то картину можно объяснить влиянием примесей фенола и белков. Большее значение оказывают примеси фенола, так как пик спектра поглощения фенола приходится на 270 нм и накладывается на пик поглощения ДНК при длине волны 260 нм.

В какой-то мере различия между разными методами можно объяснить используемыми стандартами (ДНК известной концентрации, входящей в набор). И действительно, при измерении с помощью Qubit стандарта Quantifiler (его концентрация заявлена производителем равной 200 нг/мкл) выявлена концентрация, на 5-10\% ниже заявленной. Отметим, что и Quantifiler, и Qubit в настоящее время производят в одной и той же компании - Thermo Fisher Scientific [8]. Проблема со стандартами Quantifiler была отмечена в одной из предыдущих работ. Впрочем, даже заменой используемых стандартов проблему не решить, поскольку разница в результатах методов не постоянна, а зависит как минимум от концентрации ДНК образца.

Различия в результатах методов могут зависеть и от других факторов, связанных с условиями наших экспериментов. Мы работали на ДНК, выделенной фенолхлороформным методом, в диапазоне концентраций 5-100 нг/мкл и имеющей сравнительно низкую степень фрагментации. Мы предполагаем, что обнаруженные нами закономерности простираются и на образцы ДНК, выделенной другими методами и имеющей разную степень 
сохранности, но на измерение концентраций таких образцов могут действовать дополнительные факторы, которые могли не проявиться в наших экспериментах.

Отметим, что спектрофотометрическое определение с помощью Nanodrop, не пользующееся в большинстве лабораторий репутацией надежного метода определения концентрации, в наших экспериментах зарекомендовало себя неожиданно хорошо: хотя степень погрешности и несколько выше, чем для Qubit (см. рис.), коэффициенть корреляции и с Qubit, и с Quantifiler чрезвычайно высоки (табл. 3).

Мы продемонстрировали также значение «эффекта оператора»: один и тот же метод, проведенный на одном и том же оборудовании и по одной и той же методике, но разными руками обладает разной воспроизводимостью. Это согласуется с результатами, полученными нами ранее [6].

Хотя мы не наблюдали систематической ошибки в работе двух операторов, погрешности измерений операторами с разным опытом работы значительно различаются даже в условиях тщательно проводимого эксперимента. Можно предположить, что в условиях рутинной лабораторной работы и точность измерений, и различия в точности измерений между операторами оказываются еще выше.

\section{ВЫВОДЫ}

При измерениях концентрации ДНК для одного и того же большого набора образцов, но разными методами выявлено, что значения концентрации ДНК, измеренной разными методами, хорошо коррелируют друг с другом, но значительно различаются по оцениваемой ими величине концентрации ДНК: спектрофотометрический метод (Nanodrop) дает более высокие значения концентрации, ПЦР в реальном времени (Quantifiler) - промежуточные, а фрлуориметрический (Qubit) - более низкие, причем различия могут быть двукратными. Различия в результатах разных методов более выражены для образцов с низкой концентрацией. Мы рекомендуем учитывать наличие этих систематических различий между результатами измерения концентрации ДНК, полученными разными методами. Таким образом, при указании концентрации ДНК необходимо также уточнять, каким методом и с помощью какого набора реактивов она была определена.

\section{Литература}

1. Bhat S, Curach N, Mostyn T, Bains GS, Griffiths KR, et al. Comparison of methods for accurate quantification of DNA mass concentration with traceability to the international system of units. Anal Chem. 2010 Sep 1; 82 (17): 7185-92. DOI: 10.1021/ ac100845m.

2. Li X, Wu Y, Zhang L, Cao Y, Li Y, Li J, et al. Comparison of three common DNA concentration measurement methods. Anal Biochem. 2014 Apr 15; (451): 18-24. DOI: 10.1016/j. ab.2014.01.016.

3. Nielsen K, Mogensen HS, Hedman J, Niederstätter H, Parson W, Morling N. Comparison of five DNA quantification methods. Forensic Science International: Genetics. 2008; 2 (3): 226-30.

4. He HJ, Stein EV, DeRose P, Cole KD. Limitations of methods for measuring the concentration of human genomic DNA and oligonucleotide samples. Biotechniques. 2018 Feb 1; 64 (2): 59-68. DOI: 10.2144/btn-2017-0102

\section{References}

1. Bhat S, Curach N, Mostyn T, Bains GS, Griffiths KR, et al. Comparison of methods for accurate quantification of DNA mass concentration with traceability to the international system of units. Anal Chem. 2010 Sep 1; 82 (17): 7185-92. DOI: 10.1021/ ac100845m.

2. Li X, Wu Y, Zhang L, Cao Y, Li Y, Li J, et al. Comparison of three common DNA concentration measurement methods. Anal Biochem. 2014 Apr 15; (451): 18-24. DOI: 10.1016/j. ab.2014.01.016.

3. Nielsen K, Mogensen HS, Hedman J, Niederstätter H, Parson W, Morling N. Comparison of five DNA quantification methods. Forensic Science International: Genetics. 2008; 2 (3): 226-30.

4. He HJ, Stein EV, DeRose P, Cole KD. Limitations of methods for measuring the concentration of human genomic DNA and oligonucleotide samples. Biotechniques. 2018 Feb 1; 64 (2): 59-68. DOI: 10.2144/btn-2017-0102.

5. Nakayama $Y$, Yamaguchi $H$, Einaga $N$, Esumi $M$. Pitfalls of DNA Quantification Using DNA Binding Fluorescent Dyes and Suggested Solutions. PLoS ONE. 2016; 11 (3): e0150528. DOI 10.1371/journal.pone.0150528.

6. Беленикин М. С., Галахова А. А., Балановская Е. В., Балановский О. П. Оценка воспроизводимости флуометрического измерения концентрации ДНК. Генетика. 2018; (54): 113-6. DOI: 10.1134/S0016675818130040.

7. Балановская Е. В., Жабагин М. К., Агджоян А. Т., Чухряева М. И., Маркина Н. В., Балаганская О. А. и др. Популяционные биобанки: принципы организации и перспективы применения в геногеографии и персонализированной медицине. Генетика. 2016; (12): 1371-87.

8. Qubit dsDNA BR Assay Kits Manual (MAN0002325, Revision: A.0). Available from: https://tools.thermofisher.com/content/sfs/ manuals/Qubit_dsDNA_BR_Assay_UG.pdf.

5. Nakayama $Y$, Yamaguchi $H$, Einaga N, Esumi M. Pitfalls of DNA Quantification Using DNA Binding Fluorescent Dyes and Suggested Solutions. PLOS ONE. 2016; 11 (3): e0150528. DOI 10.1371/journal.pone.0150528.

6. Belenikin MS, Galahova AA, Balanovskaya EV, Balanovsky OP. Ocenka vosproizvodimosti fluometricheskogo izmereniya koncentracii DNK. Genetika. 2018; (54): 113-6. DOI: 10.1134/ S0016675818130040. Russian.

7. Balanovskaya EV, Zhabagin MK, Agdzhoyan AT, Chukhryaeva MI, Markina NV, Balaganskaya OA, et al. Population biobanks: Organizational models and prospects of application in gene geography and personalized medicine. Russian Journal of Genetics. 2016; 52 (12): 1227-43.

8. Qubit dsDNA BR Assay Kits Manual (MAN0002325, Revision: A.0). Available from: https://tools.thermofisher.com/content/sfs/ manuals/Qubit_dsDNA_BR_Assay_UG.pdf. 